\title{
On the spatial structure of global linear instabilities and their experimental identification *
}

\author{
Vassilios Theofilis ${ }^{\mathrm{a}, 1}$ \\ a DLR Institute of Fluid Mechanics, Division of Transition and Turbulence, Bunsenstraße 10, 37073 Göttingen, Germany
}

\begin{abstract}
The purpose of the present paper is to discuss the spatial structure of global instabilities, solutions of the partial derivative eigenvalue problem resulting from a nonparallel linear instability analysis of the incompressible Navier-Stokes and continuity equations, as developing upon four prototype essentially twodimensional steady laminar flows. Theoretical knowledge of these eigendisturbances is instrumental to devising measurement techniques appropriate for their experimental recovery and ultimate control of laminarturbulent transition mechanisms. Raising the awareness of the global linear flow eigenmodes contributes to redefining the boundaries between experimental observations which may be attributed to linear global as opposed to nonlinear mechanisms.
\end{abstract}

laminar-turbulent flow transition / nonparallel linear instability / partial-derivative eigenvalue problem

Zusammenfassung

Über die räumliche Struktur globaler linearer Instabilitäten und deren experimenteller Erkennung. Zweck dieses Artikels ist eine Diskussion der räumlichen Struktur globaler Instabilitäten, die von einer nichtparallelen linearen Instabilitätsanalyse der inkompressiblen Navier-Stokeschen und Kontinuitätsgleichungen mittels Lösungen eines partiellen Differentialgleichungs-Eigenwertproblems gewonnen werden. Dazu wurden vier prototype Konfigurationen gewählt, die essentielen zweidimensionalen stationären laminaren Strömungen entsprechen. Theoretische Kentnisse über diese Eigenstörungen ist eine Grundvoraussetzung für die Konstruktion experimenteller Methoden, um die globalen Instabilitäten messen zu können und zu steuern. Eine erhöhte Wahrnehmung der globalen linearen Strömungsmoden trägt dazu bei, die Grenzen zwischen experimentellen Beobachtungen, die als lineare globale bzw. nichtlineare Phenomäne anerkannt werden, neu zu definieren.

laminare-turbulente Transition von Strömungen / nichtparallele lineare Instabilität / partielles Differentialgleichungs-Eigenwertproblem

Nomenclature
$\begin{array}{lll} & \mathcal{D}_{x}^{2} & \partial^{2} / \partial x^{2} \\ \text { Latin Symbols } & \mathcal{D}_{y} & \partial / \partial y \\ \text { A } \quad \text { aspect ratio } & \mathcal{D}_{y}^{2} & \partial^{2} / \partial y^{2} \\ \mathcal{D}_{x} \quad \partial / \partial x & \mathrm{i} & \text { imaginary unit }\end{array}$

* This paper is based on the DLR Zentrumskolloquium entitled "On the origins of transition and turbulence in three-dimensional flows" delivered on 15 April 1999 at the Institute of Fluid Mechanics of the DLR Göttingen

${ }^{1}$ E-mail: Vassilios. Theofilis@dlr.de 


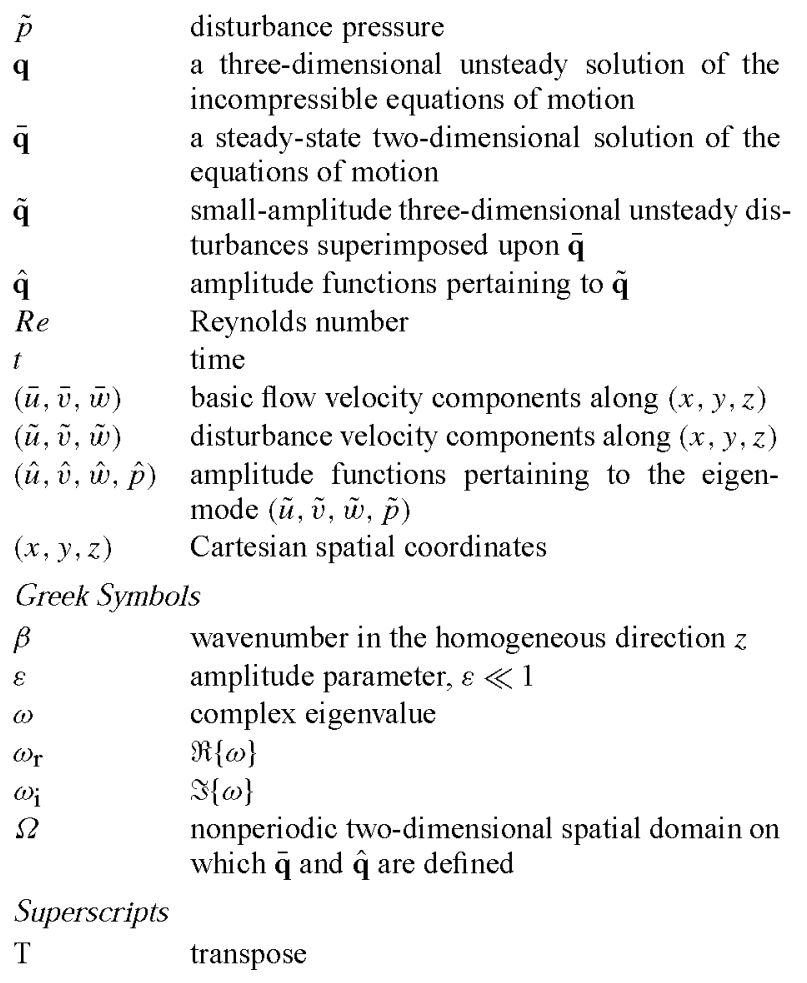

\section{Introduction}

The quest for identification of deterministic routes in the phenomenon of laminar-turbulent fluid flow transition has been pursued, for the best part of last century, through numerical solution of the linear eigenvalue problem proposed by Tollmien [26]. The premises on which Tollmien's theory is based is the decomposition of any flow quantity into a steady one-dimensional profile and an unsteady three-dimensional part, superimposed upon the steady so-called basic flow at a linearly small amplitude $\varepsilon$. The resulting system of equations at $\mathrm{O}(1)$ describes the basic state (e.g. the Blasius profile in the incompressible flat-plate boundary layer or the parabolic profile of plane Poiseuille flow), the system at $O(\varepsilon)$ is the one solved while that at $O\left(\varepsilon^{2}\right)$ is neglected. Separability of the linearised system of equations is achieved if, firstly, two out of the three spatial directions are considered as homogeneous and treated as periodic and, secondly, the basic flow velocity component in the spatial direction normal to the wall in a boundary-layer type of flow is neglected. The latter so-called 'parallel-flow' assumption severely constrains the type and number of flows on which Tollmien's classic linear instability theory may be applied and, even within the realm of its applicability, has led to a considerable amount of controversy in the past. The disturbance components along the homogeneous spatial directions are decomposed in multiples of fundamental wavenumbers in each spatial direction. The third spatial direction, the same as that on which the basic flow depends, is resolved. The system at $O(\varepsilon)$ in incompressible flow is none other than that which gives rise to the Orr-Sommerfeld and Squire equations [6]. The linear instabilities in physical space may then be interpreted as plane or oblique wave-like disturbances, depending on whether a non-zero fundamental wavenumber is considered in one or two spatial directions, respectively. Following the celebrated experiment of Schubauer and Skramstadt [21], who demonstrated the existence and linear amplification of wave-like disturbances (what later became known as the Tollmien-Schlichting instability waves) prior to laminar-turbulent breakdown in the incompressible flat-plate boundary-layer flow, intense exploitation of Tollmien's linear theory and its extensions to compressible flow [13] has created a wealth of information regarding the transition phenomenon on account of this type of linear instability mechanism [16].

A widespread misconception in the community is that linear instability is associated with wave-like disturbances alone. Consequently, experimental work aiming at the identification of linear instability mechanisms is currently almost exclusively utilising single hot-wire probes which are transversed along a single spatial direction (e.g. the wall-normal direction in a boundary-layer type of flow) in order for information on the spatial structure of the disturbances to be collected. Such an approach implicitly assumes that the only linear disturbances in the flow are of wave-like nature and the aim of the experimentation is to identify the spatial structure of the amplitude functions of different instability modes. Clearly the success of such an approach is limited by its inherent assumptions; one either has no means of identifying structures in the flow other than the ones aimed at, or if indeed identified, global linear instabilities which exist in the flow may well be confused with nonlinear flow behaviour.

On account of the property of resolution of a single spatial direction we term Tollmien's linear instability theory a 'one-dimensional' theory. Potential of confusion is immediately generated with both theoretical and experimental work in the literature, which terms the case of existence of a nonzero wavenumber along one of the two aforementioned homogeneous spatial directions as a 'two-dimensional' and that in which nonzero wavenumbers along both periodic spatial directions exist as a 'three-dimensional' linear instability (e.g. [2,20]); in our terminology both are approaches aiming at the quantification of one-dimensional disturbances. Within the framework of the parabolised stability equations (PSE), on the other hand, one resolves one spatial direction and considers only mild dependence of the basic flow and the instabilities on a second spatial direction (see [10] and references therein); the third spatial direction is again taken to be periodic. Such a treatment of the instability problem could be termed a quasi-two-dimensional approach. 
Here we are concerned with essentially two-dimensional linear instabilities. In a manner analogous to Tollmien's theory, the two-dimensional (also herein referred to as global) linear instability analysis is concerned with the decomposition of a flow quantity into steady basic and unsteady disturbance fields, the latter superimposed at a small amplitude $\varepsilon$ upon the former. However, both the basic and the disturbance quantities are two-dimensional functions of two resolved spatial directions. The basic flow is again obtained at $\mathrm{O}(1)$ and the $\mathrm{O}\left(\varepsilon^{2}\right)$ terms are neglected; the $O(\varepsilon)$ system of linear equations, on the other hand, has coefficients which are independent of one spatial direction and time and, as such, may be written in the form of a complex nonsymmetric linear partial derivative eigenvalue problem. In incompressible flow the only free parameter of the problem, besides the Reynolds number, is the wavenumber of the disturbances in the single periodic spatial direction. The solution of a system of partial differential equations in two-dimensional linear instability analysis, as opposed to the system of ordinary differential equations that the one-dimensional theory addresses, offers a great deal of more freedom in the choice of boundary conditions associated with the problem of global linear instability and, hence, has obvious consequences for the character of the solutions obtained.

The purpose of the present paper is to discuss some such structures with the aid of four examples of flow in both closed and open systems, under a variety of boundary conditions imposed by the flow physics. We discuss the eigenvectors delivered by the partial derivative eigenvalue problem analysis of global linear instability in the rectangular duct, the infinite-aspect-ratio limit of which is plane Poiseuille flow, the lid-driven cavity for which no rational approximation exists which may reduce the linear instability problem to one for which the one-dimensional theory may be used (except, perhaps, in the large aspect-ratio limit of the flow), boundarylayer flow incorporating a laminar separation bubble for which plenty of work exists using one-dimensional analysis but virtually none using two-dimensional linear theory and, finally, the infinite swept attachmentline boundary layer formed on the windward face of swept cylinders and wings. The latter problem is one in which experimentation on the basis of the assumptions of the one-dimensional theory, in conjunction with a particular wall-normal structure of the global linear instabilities has resulted in the latter having remained concealed in the results of continuous experimentation over four decades. Both the numerical aspects and detailed presentation of the results obtained from a physical point of view are discussed elsewhere. In the limited space available here the focus is placed on exposure of the spatial structure of the eigenvectors in an attempt to raise the awareness of the community, especially of experimentalists, with respect to the expected linear disturbances potentially to be found in a given flow problem. In section 2 we briefly discuss the partial derivative eigenvalue problem theory before presenting in section 3 results for the most significant, from a linear instability analysis point of view, eigenmodes in the aforementioned examples as well as their spatial structure. Closing remarks are presented in section 4 .

\section{The partial-derivative eigenvalue problem theory}

Alternative formulations exist which address the partial-derivative eigenvalue problem governing global (two-dimensional) linear flow instability. Here we restrict ourselves to the presentation of the most general, primitive-variables formulation, for the sake of introduction of the necessary notation. Taking incompressible flow, a solution of the equations of motion, $\mathbf{q}=$ $(u, v, w, p)^{\mathrm{T}}$, may be considered as composed of smallamplitude three-dimensional perturbations $\tilde{\mathbf{q}}=(\tilde{u}, \tilde{v}, \tilde{w}$, $\tilde{p})^{\mathrm{T}}$ superimposed upon a steady two-dimensional basic flow $\overline{\mathbf{q}}=(\bar{u}, \bar{v}, \bar{w}, \bar{p})^{\mathrm{T}}$ according to

$$
\mathbf{q}(x, y, z, t)=\overline{\mathbf{q}}(x, y)+\varepsilon \tilde{\mathbf{q}}(x, y, z, t),
$$

with $\varepsilon \ll 1$. Here the three spatial directions are denoted by $x, y$, and $z$, respectively and $t$ denotes time. Linearisation about $\overline{\mathbf{q}}$ is permissible on account of the smallness of perturbations compared with the steady-state $\overline{\mathbf{q}}$ and the resulting system for the determination of $\tilde{\mathbf{q}}$ is separable in both $t$ and $z$ on account of the steadiness and the twodimensionality of the basic flow $\overline{\mathbf{q}}$. Eigenmodes are introduced in these directions such that

$$
\tilde{\mathbf{q}}(x, y, z, t)=\hat{\mathbf{q}}(x, y) \mathrm{e}^{\mathrm{i}[\beta z-\omega t]}+c . c .
$$

with $\hat{\mathbf{q}}=(\hat{u}, \hat{v}, \hat{w}, \hat{p})^{\mathrm{T}}$ containing the perturbation velocity components and disturbance pressure amplitude functions and $i=\sqrt{-1}$. Complex conjugation is introduced in (2) since $\tilde{\mathbf{q}}$ is real while all three of $\hat{\mathbf{q}}, \beta$ and $\omega$ may be complex. In the framework of the temporal linear nonparallel instability analysis used presently we write the linearised system in the form of an eigenvalue problem for $\omega$, while $\beta$ is taken to be a real wavenumber parameter describing an eigenmode in the $z$-direction. The real part of $\omega$ is related with the frequency of the instability mode while its imaginary part is the growth/damping rate; a positive value of $\omega_{\mathrm{i}} \equiv \Im\{\omega\}$ indicates exponential growth of $\tilde{\mathbf{q}}$ in time $t$ while $\omega_{\mathrm{i}}<0$ denotes decay of $\tilde{\mathbf{q}}$ in time. In the present framework the three-dimensional space comprises an arbitrary two-dimensional domain $\Omega$ extended periodically in $z$ and characterised by a wavelength $L_{z}$ in this direction, which is in turn associated with the wavenumber of each eigenmode, $\beta$, through $L_{z}=2 \pi / \beta$. The system for the determination of $\omega$ and $\hat{\mathbf{q}}$ takes the form of a complex nonsymmetric generalised eigenvalue problem 


$$
\begin{aligned}
{\left[\mathcal{L}-\left(\mathcal{D}_{x} \bar{u}\right)\right] \hat{u}-\left(\mathcal{D}_{y} \bar{u}\right) \hat{v}-\mathcal{D}_{x} \hat{p} } & =-\mathrm{i} \omega \hat{u}, \\
-\left(\mathcal{D}_{x} \bar{v}\right) \hat{u}+\left[\mathcal{L}-\left(\mathcal{D}_{y} \bar{v}\right)\right] \hat{v}-\mathcal{D}_{y} \hat{p} & =-\mathrm{i} \omega \hat{v}, \\
-\left(\mathcal{D}_{x} \bar{w}\right) \hat{u}-\left(\mathcal{D}_{y} \bar{w}\right) \hat{v}+\mathcal{L} \hat{w}-\mathrm{i} \beta \hat{p} & =-\mathrm{i} \omega \hat{w}, \\
\mathcal{D}_{x} \hat{u}+\mathcal{D}_{y} \hat{v}+\mathrm{i} \beta \hat{w} & =0,
\end{aligned}
$$

subject to appropriate boundary conditions on $\partial \Omega$. The linear operator $\mathcal{L}=(1 / R e)\left(\mathcal{D}_{x}^{2}+\mathcal{D}_{y}^{2}-\beta^{2}\right)-\bar{u} \mathcal{D}_{x}-$
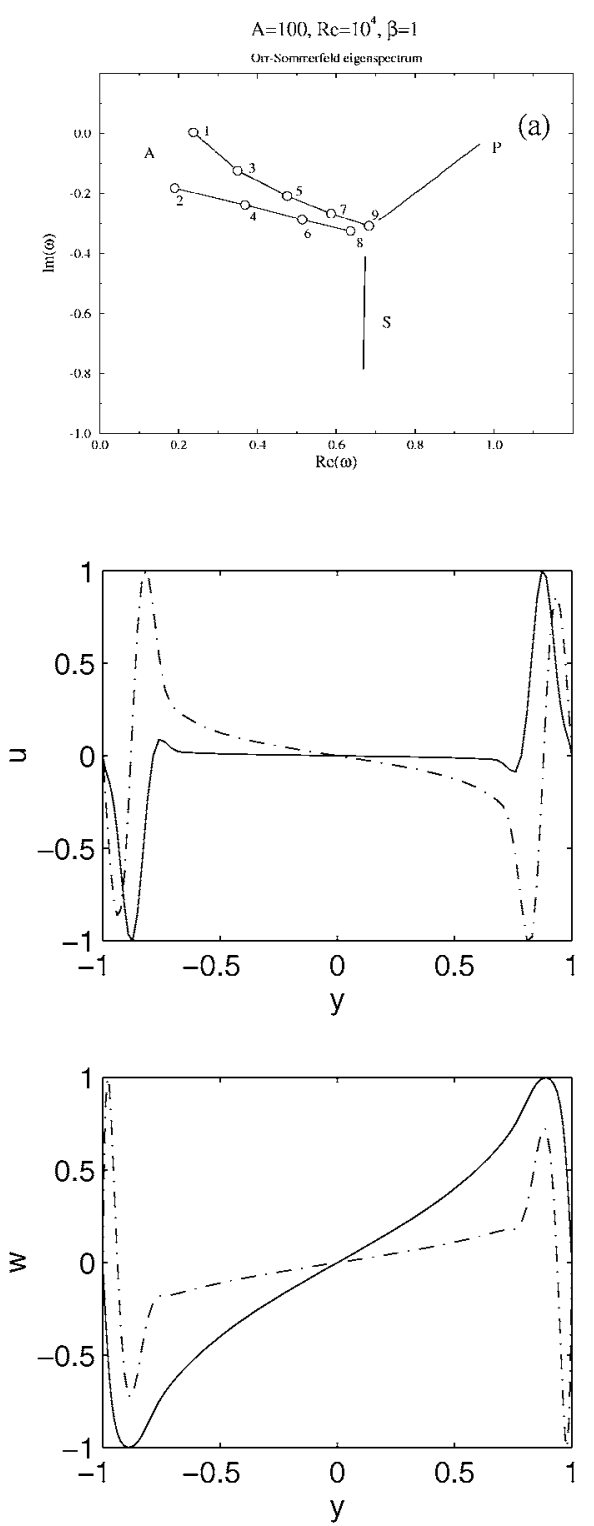

$\bar{v} \mathcal{D}_{y}-\mathbf{i} \beta \bar{w}$. Some details on the accurate and efficient numerical solution of the partial-derivative eigenvalue problem (3)-(6) have been presented by Theofilis [24]. The reader's attention is drawn to the boundary conditions associated with this system. This issue is straightforwardly resolved in the one-dimensional limit of (3) (6) where one imposes homogeneous Dirichlet boundary conditions on the disturbance velocity components on both solid boundaries and the free-stream. The reso-
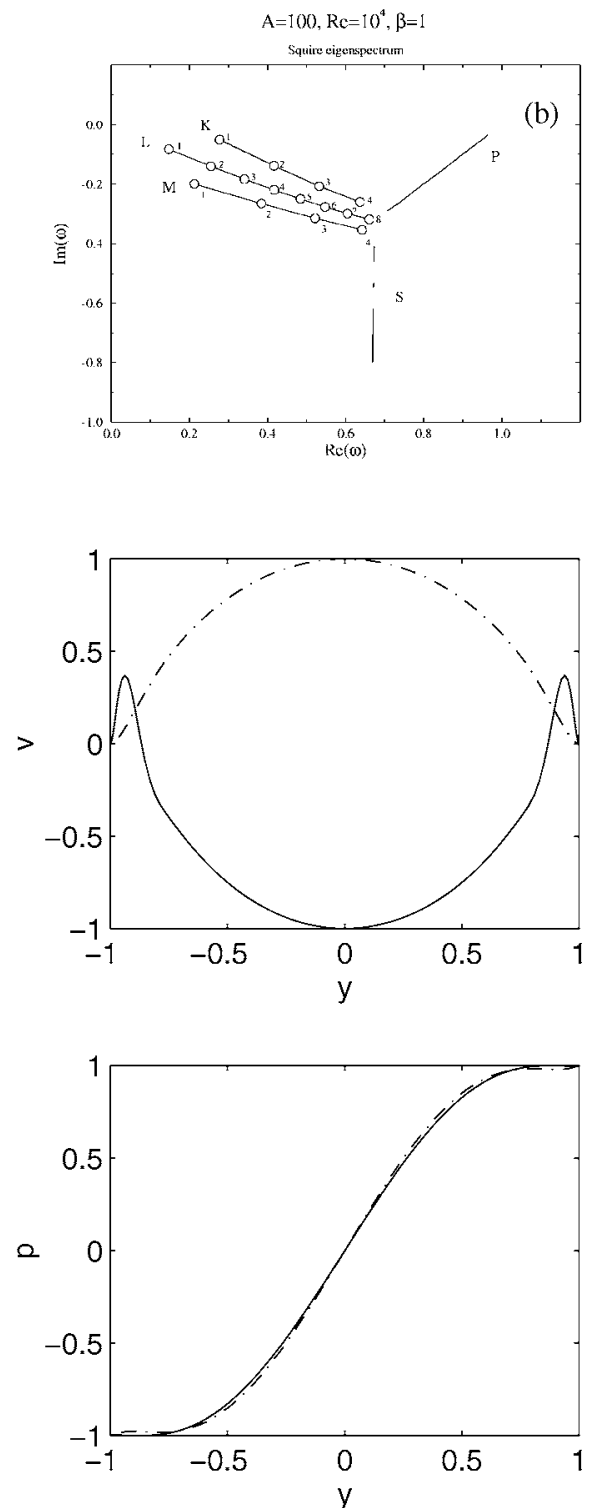

Figure 1. Upper: the Orr-Sommerfeld and Squire spectra obtained by numerical solution of the partial-derivative eigenvalue problem in the rectangular duct at $R e=10^{4}, \beta=1, A=100$. In both cases $\mathrm{P}$ and $\mathrm{S}$ correspond to the continuous spectrum; the Orr-Sommerfeld discrete eigenspectrum is denoted by A [15] and that of the Squire modes falls into three families, denoted by K, L and M. Lower: the disturbance eigenfunctions corresponding to the unstable mode; solid lines denote the real and dashed-dotted the imaginary parts of the eigenfunctions. 
lution of two spatial directions, $x$ and $y$ in (3)-(6), on the other hand results in an elliptic problem for which boundary conditions on $\partial \Omega$ are required. Here intuition offered by the flow physics alone may provide plausible answers. We discuss in what follows both closed and open problems, as examples of successful resolution of the issue of boundary conditions associated with the partial derivative eigenvalue problem.

Regarding the numerical methods for the solution (3)(6), briefly, application of the classic QZ algorithm for the recovery of the full spectrum of eigenvalues/eigenvectors of the partial derivative eigenvalue problem is not advisable on account of both the memory and runtime requirements of this algorithm which scales with the square and the cube, respectively, of the product of the number of points resolving each of the spatial directions $x$ and $y$. Krylov subspace iteration, on the other hand, offers an efficient means of recovering the most interesting, from an instability analysis point of view, part of the eigen- spectrum at a fraction of the cost of the QZ algorithm. This permits probing into flow instability at substantially higher Reynolds numbers at a given level of computing cost, compared with the QZ.

\section{Results}

\subsection{Flow in a rectangular duct}

A minimal test to which a new extension of an established theory must be subjected is the reproduction by the former of the results of the latter theory. The rectangular duct flow is well suited for this exercise, since its large aspect ratio may be described by the classic plane Poiseuille flow (PPF), the linear instability results of which are well-known [18]. Tatsumi and Yoshimura [22], who were the first to address the problem of nonparallel linear instability in the rectangular duct albeit using different numerical methods and more restrictive
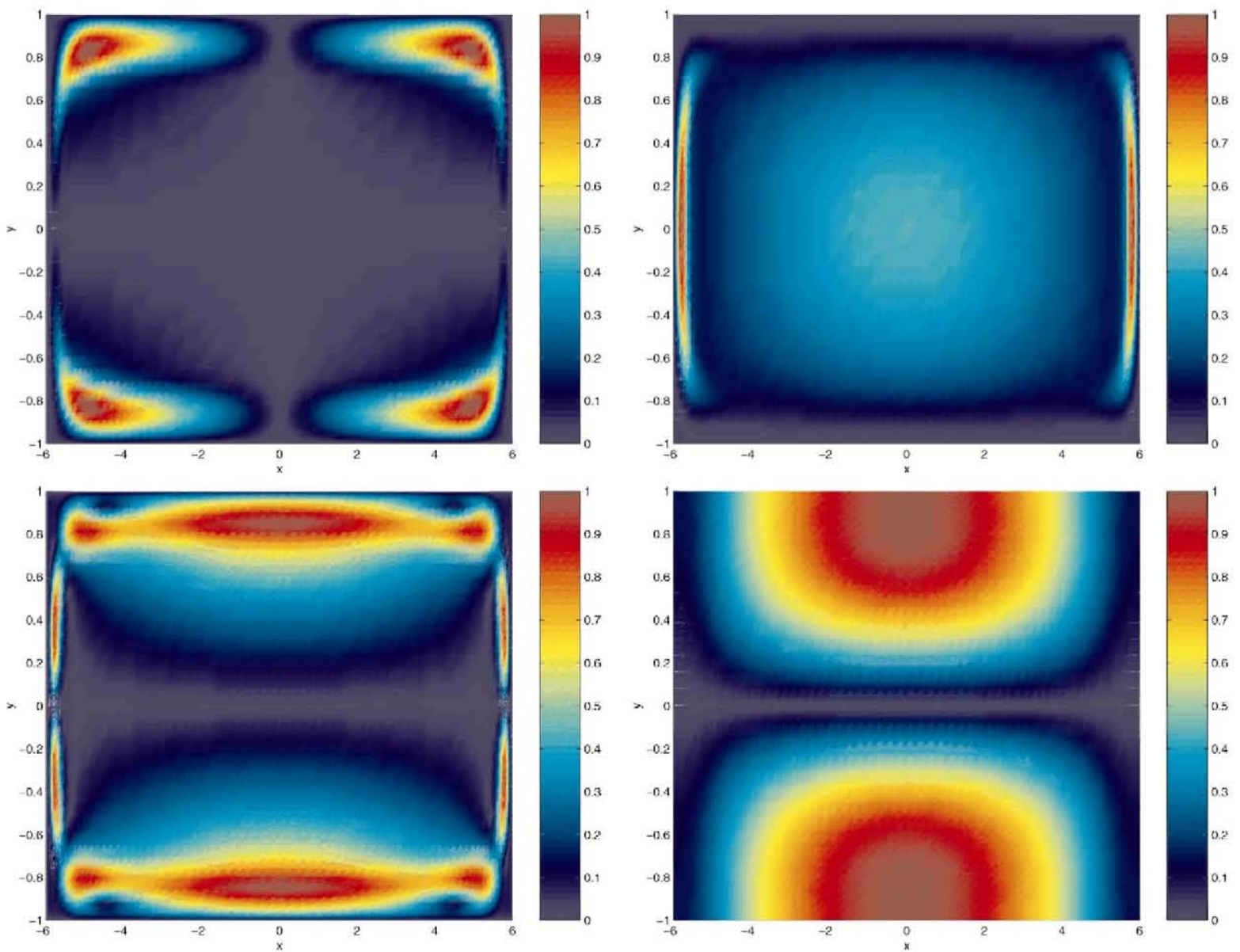

Figure 2. The spatial structure of the neutral wall mode in rectangular duct flow at $R e=7500, \beta=1, A=6$ : normalised magnitude of the disturbance (top left) velocity component $\hat{u}$; (top right) velocity component $\hat{v}$; (bottom left) velocity component $\hat{w}$; (bottom right) pressure $\hat{p}$. 

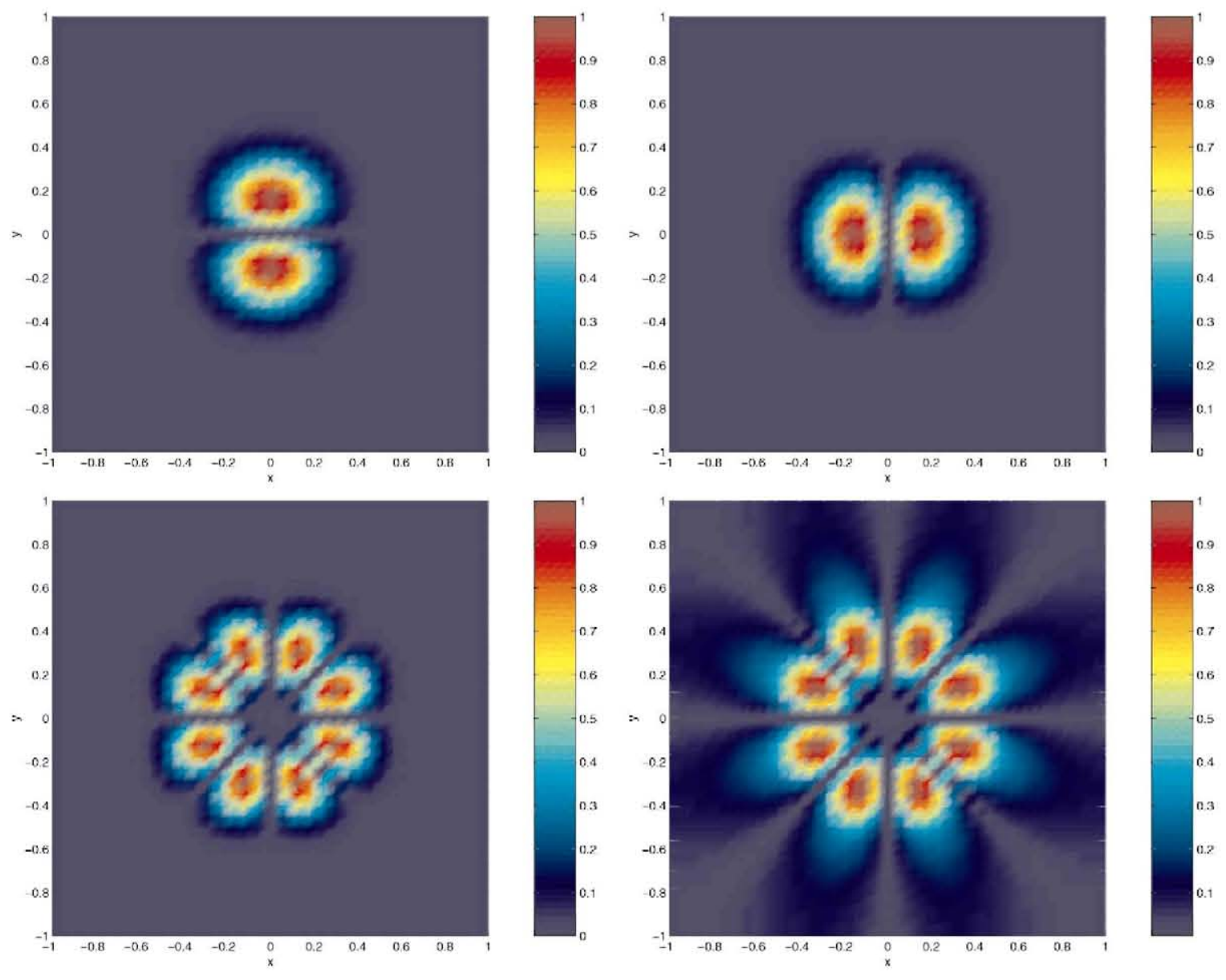

Figure 3. The spatial structure of a decaying centre mode in rectangular duct flow at $R e=4000, \beta=1$ : normalised magnitude of the disturbance (top left) velocity component $\hat{u}$; (top right) velocity component $\hat{v}$; (bottom left) velocity component $\hat{w}$; (bottom right) pressure $\hat{p}$.

boundary conditions compared with those used presently, have mentioned that they also subjected their algorithm to the same test by explicitly neglecting the dependence of the problem on one spatial dimension. By contrast here we let $A \rightarrow \infty$, in practice permitting the aspect ratio to take large finite values. In a series of careful experiments Nishioka and co-workers (e.g. [17]) have established that an aspect ratio in excess of $A=25$ is necessary in order for the PPF instability results to be recovered experimentally; a value $A=100$ is taken presently. Calculations have been performed at the parameters of the test case of Orszag [18], $R e=10^{4}, \beta=1$ using $N y=48$ and a variable number of $N x$ collocation points resolving the $y \in[-1,1]$ and $x \in[-A, A]$ directions respectively. As the aspect ratio increases, the eigenvalue spectrum is found to be composed of clusters of $N x$ copies of the PPF Orr-Sommerfeld and Squire spectra, recovered to within six significant digits. The spectra are presented in figure 1 clustered into families of eigenmodes [15]; the eigenfunctions corresponding to the most unstable mode are also shown in this figure.

The aspect ratio is subsequently decreased to $O(1)$ values and the results of Tatsumi and Yoshimura [22] are fully recovered [24]. From the point of view of the theme of the present paper, the spatial structure of the disturbance velocity components pertaining to the critical wall mode at $A=6$ are shown in figure 2. Parameters used for this calculation are $R e=7500, \beta=1$; the calculated eigenvalue is $\omega=(0.2542,0.0)$. Several aspects of these results deserve discussion. The symmetries present in these results were imposed by Tatsumi and Yoshimura [22] for reasons of numerical efficiency; by contrast we recover these symmetries as a result of our calculations. Taking cuts through the line of symmetry $x=0$ delivers a $y$-distribution of the eigenmodes which is very similar to that found in the one-dimensional limit of the analysis discussed in figure 1; this fact is particularly evident in the results for the $\hat{v}, \hat{w}$ and $\hat{p}$ distur- 


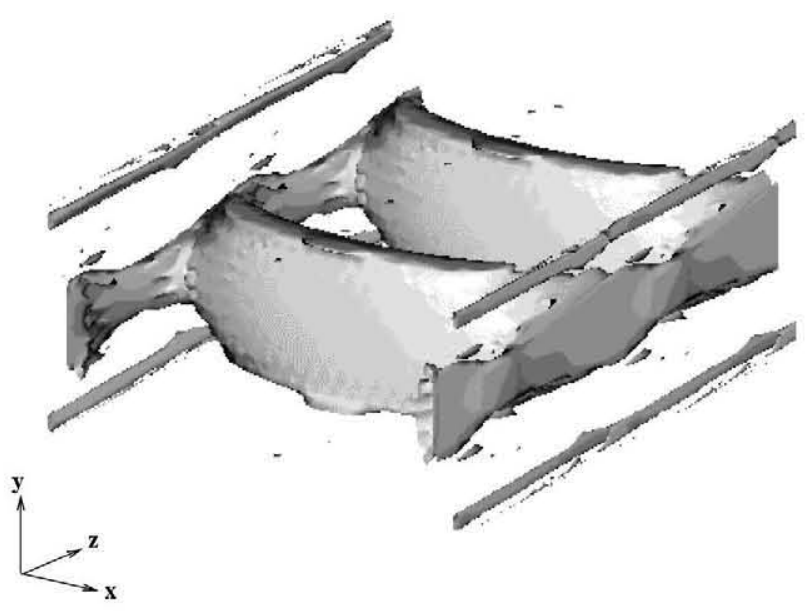

Figure 4. Isosurface of the disturbance vorticity magnitude of the neutral wall mode pertaining to flow in an $A=6$ duct at $\operatorname{Re}=7500, \beta=1$.

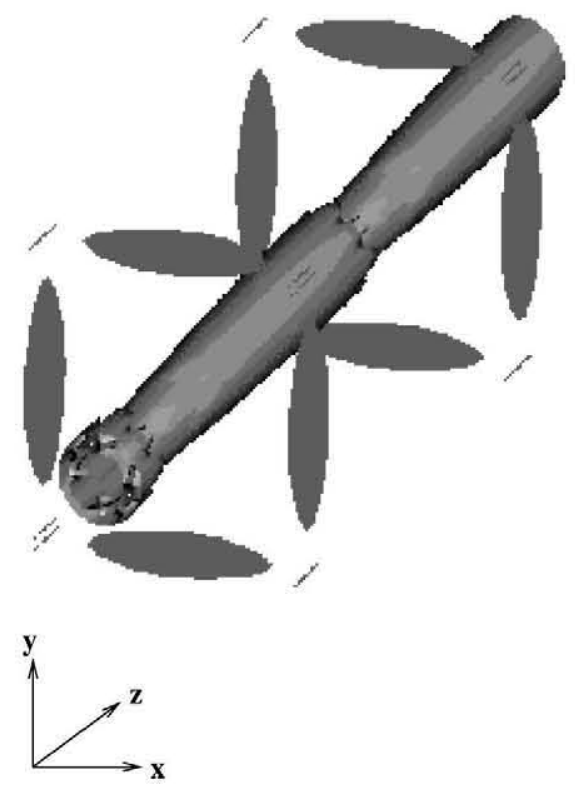

Figure 5. Isosurface of the disturbance vorticity magnitude of a decaying centre mode pertaining to flow in a square duct at $\operatorname{Re}=4000, \beta=1$.

bances. However, the most striking feature of the results is the pronounced wall-effects which are inaccessible to the one-dimensional theory. Elaborating on the last point, we mention that the scope of application of the traditional approach of transversing hot-wire probes through the flowfield in order for global two-dimensional linear eigenfunctions of the form presented in the results of figure 2 to be recovered is very limited indeed. In order for such results to be recovered reliably the experimental approach used must be based on global field measurements, capable of recording the flowfield simultaneously at several different times; the decomposition (1)-(2) may then be used in order to calculate the amplitude functions $\hat{\mathbf{q}}$. An analogous conclusion may be drawn by inspection of the centre modes pertaining to flow in the square duct, a typical example of which at $R e=4000, \beta=1$ having an eigenvalue $\omega=(0.959,-0.041)$ is presented in figure 3 . A further point worthy of discussion here is that knowledge of the spatial structure of the two-dimensional linear eigenmodes limits the possibility of confusing such global coherent structures with nonlinear flow behaviour. This point may be strengthened by viewing isosurfaces of the magnitude of the disturbance vorticity pertaining to the results presented, which are presented in figures 4 and 5; arbitrary scales have been used for the present linear results. Needless to say, such structures manifest themselves as global flowfield oscillations without any evidence of travelling wave-like behaviour.

\subsection{The lid-driven cavity}

Another closed-system flow problem in which linear instability analysis may only be performed in the present nonparallel linear analysis framework is that of a flow developing in the square lid-driven cavity. Here no simplifications exist which may reduce the instability analysis to a (one-dimensional) ordinary-differential-equation-based eigenvalue problem and one has to resort to the twodimensional partial-derivative eigenvalue problem or, alternatively, to direct numerical simulation [12]. Short of following the latter path, which is not well-suited for parametric studies and in which different instability mechanisms might be confused, we solve the eigenvalue problem (3)-(6) at given real parameters $\beta$ in order to recover the frequency $\omega_{\mathrm{r}}$ and the growth/damping rate $\omega_{\mathrm{i}}$ of the respective eigenmode. Alongside, we recover the spatial structure of the eigenfunctions pertaining to each mode. In line with the spirit of the present contribution we refrain from discussing either numerical aspects or the open issue of the critical Rey nolds number in this flow $[4,19]$ and concentrate on the qualitative discussion of the spatial structure of the eigenfunctions and of the flowfield which is set up by linear superposition of the two most unstable three-dimensional $(\beta \neq 0)$ eigenmodes upon the steady basic flow. The aim here is to demonstrate that a) linear mechanisms are indeed at play in the nonparallel laminar steady basic flow which b) have nothing to do with the classic wave-like structures encompassed in Tollmien's theory.

Figure 6 (left) shows a perspective view of the spatial distribution of the least-stable stationary eigenmode at a low Reynolds number, $R e=100$ and a wavenumber $\beta=1$. In figure 6 (right) the next in significance travelling eigenmode is presented. In all plots the lower left corner of the domain corresponds to that of the cavity and the origin of the axes $(0,0)$, with the lid defined at $x \in[0,1], y=1$; the upper row shows the real parts of the eigendisturbances, the lower row the corresponding imaginary parts, and the first to fourth column contains 

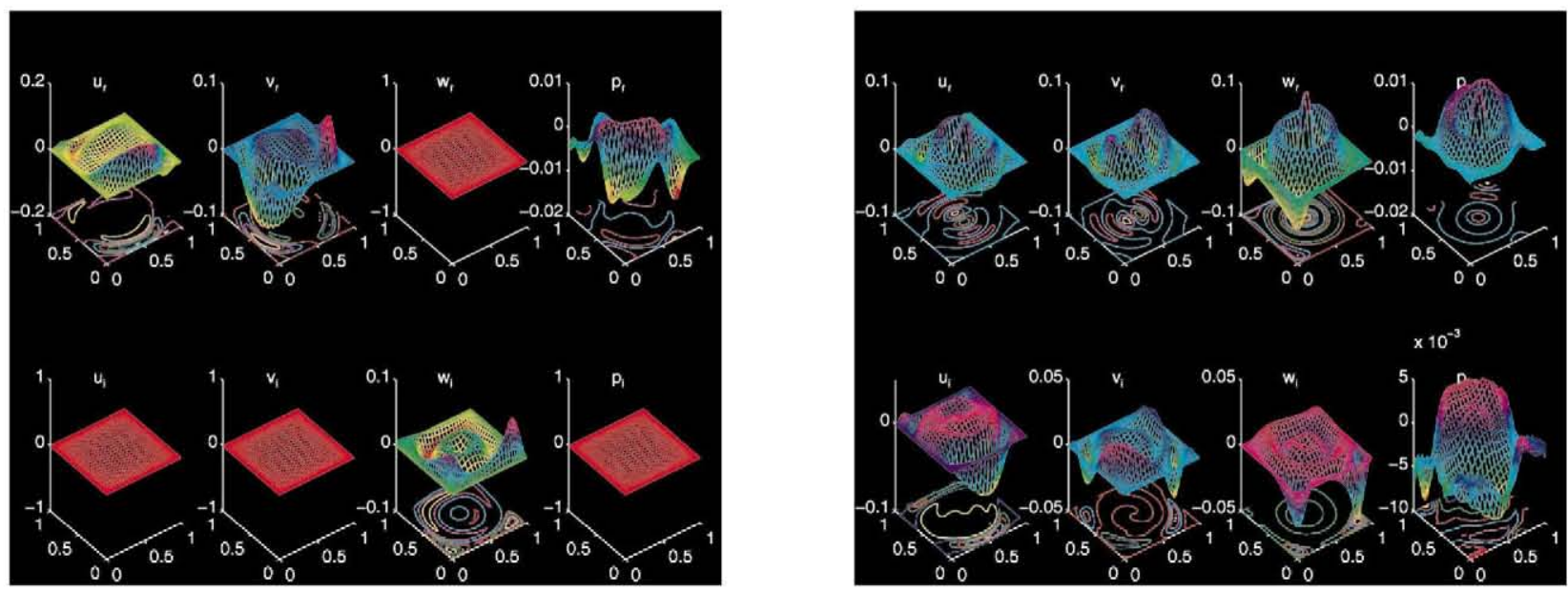

Figure 6. Contour lines of the least-stable (damped) stationary mode (left), and travelling mode (right) in a square lid-driven cavity at $\operatorname{Re}=100, \beta=1$. Shown is the two-dimensional eigenvector $(\hat{u}, \hat{v}, \hat{w}, \hat{p})^{\mathrm{T}}$ as a function of the cavity coordinates $(x \in[0,1] \times y \in$ $[0,1])$.

the eigenvectors $(\hat{u}, \hat{v}, \hat{w}, \hat{p})^{\mathrm{T}}$. Arbitrary scales have been used for the present linear results. Qualitative analogies between the results of the two figures exist with respect to the following characteristics. First, the (damped at these parameters) linear eigenmode is shown to inherit the general features of the basic flow to which it corresponds; the flowfield set up by the $x$-wise velocity component is in the direction of the lid motion in the upper half and in the opposite direction in the lower half of the cavity. Analogously, the disturbance velocity component in the $y$-direction is such that fluid flows in the positive sense along this axis in $y<0.5$ and in the opposite sense in $y>0.5$. The disturbance velocity component in the third spatial direction $z$ provides a clear view of the vortical motion set up in the cavity. Finally, the disturbance pressure has been found to be the hardest eigenfunction to resolve; its correct capturing is key to reliability of the partial-derivative eigenvalue problem results. In a manner analogous to the one-dimensional linear instability analysis theory, the higher the eigenmode the more complicated its structure, as can clearly be seen by comparing the results of the figure 6 .

At a much higher Reynolds number $R e=1000$, predicted by Ding and Kawahara [4] to lie within the neutral loop of this flow, the first travelling eigenmode generates a rather complicated flowfield, which has been visualised by means of isocontours of the spanwise disturbance velocity component $\hat{w}$ and shown at an arbitrarily-defined amplitude in figure 7 . For simplicity only one of the complex conjugate pair of eigenmodes is presented; in the flow a linear superposition of both, forming a standing-wave pattern, is to be expected. The edges $(0,0)$ and $(1,1)$ of the cavity correspond to the lower left and upper right corners in this figure, respectively, and one periodicity length is shown in the third (spanwise) spatial direction. One may appreciate in these results the complexity of the flowfield set up by the linear mechanism discussed here; well-defined structures exist adjacent to all three stationary walls, $x=0, y=0$ and $x=1$ while disconnected structures, all pertaining to the same linear eigenmode are to be found in the centre of the cavity. The point to be made with the aid of these results is, again, twofold. Firstly, any attempt to recover such structures by single-probe transversing is doomed to failure; global simultaneous field measurements are necessary for structures of the kind shown in figure 7 to be identified. Second, power spectral analysis of the flowfield at conditions favouring growth of several eigenmodes makes identification of the latter in three-dimensional physical space a rather laborious task. In such cases it is advisable to focus on slightly supercritical conditions in the neighbourhood of the neutral loop in order to isolate and identify individual eigenmodes. In this respect, prior theoretical information based on the numerical solution of the partial-derivative eigenvalue problem (3)-(6) is indispensable.

\subsection{The laminar separation bubble}

A flow whose linear instability still entails many open questions, despite the large amount of knowledge having been amassed over decades of research [5], is that in a boundary layer which encompasses a closed recirculation zone generated by an adverse pressure gradient, such as that depicted in figure 8 . A detailed discussion of the different instability mechanisms operative in this flow may be found in [25]; an analogous global linear instability problem has been recently studied in separated-flow set up by the backward-facing step geometry [1]. The major difference, from a numerical point of view, with both 


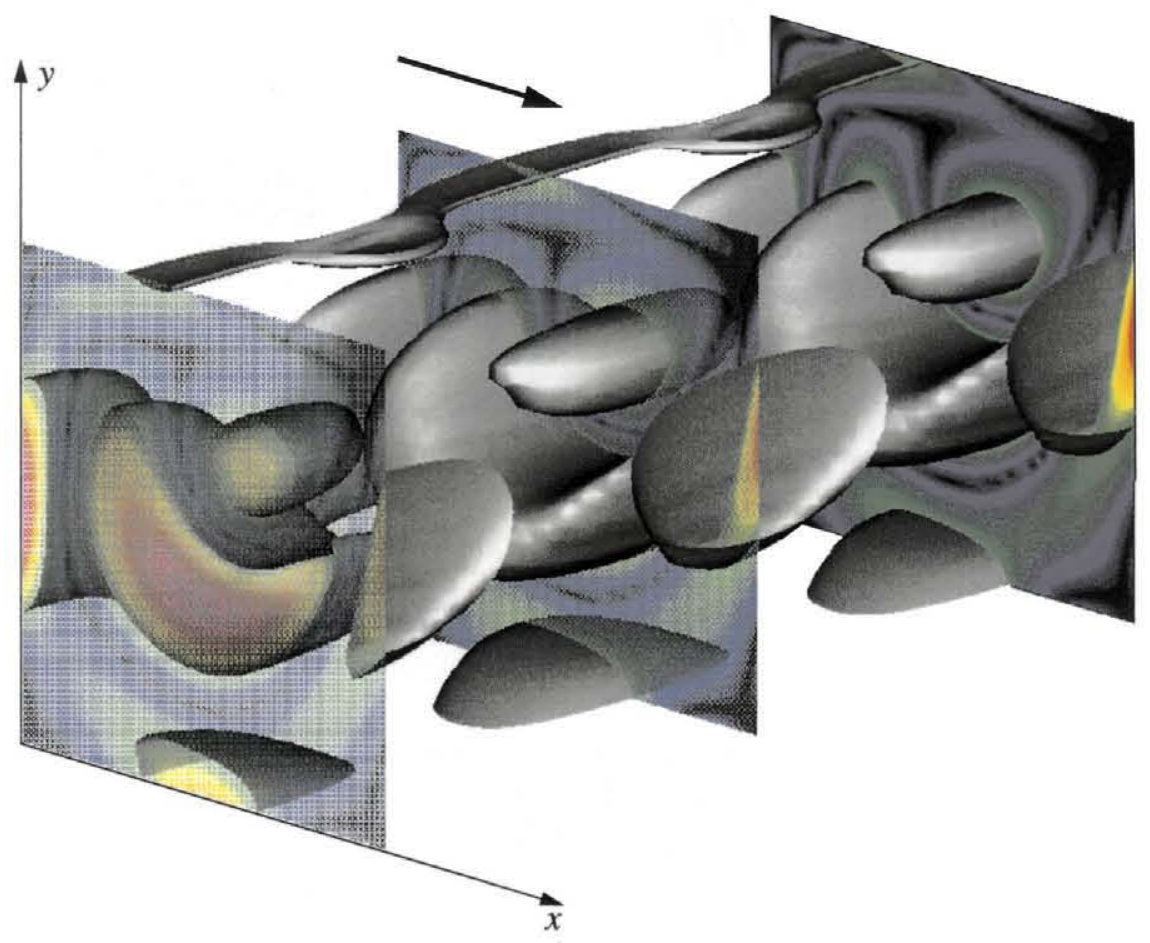

Figure 7. Isosurface of the spanwise disturbance velocity component of the most unstable mode at $\operatorname{Re}=1000, \beta=7.5$. Flow is in the direction of the arrow.

flows of sections 3.1 and 3.2 concerns the boundary conditions for the elliptic partial-derivative eigenvalue problem (3)-(6). Flow in closed domains may be tackled using the usual viscous boundary conditions for the disturbance velocity components alongside compatibility conditions for the disturbance pressure to close the system of equations. In the case of an open flow system, on the other hand, the issue of boundary conditions must be considered carefully with respect to the plausible physical situations that one wishes to model. We have addressed this problem by imposing homogeneous Dirichlet conditions on all perturbations at inflow, thus prohibiting any disturbances to enter the calculation domain and allowing only those generated by the presence of the steady laminar separation bubble. Extrapolation of information from the interior of the integration domain was used at outflow. On the wall and at the far-field the usual viscous boundary conditions are employed.

The implications on unsteadiness of the steady laminar flow deriving from linear amplification of the global eigenmode discovered theoretically by [25] to grow linearly upon the two-dimensional laminar basic flow of [3] at $R e=10^{6} / 48$ have been discussed extensively in [25]. There we also discussed one scenario potentially explaining the three-dimensionalisation of the reattachmentline as observed in experiment. Here, we present in figure 9 in perspective view colour-coded contours of the wall-normal disturbance velocity component $\hat{v}$, as well as isosurfaces of the (actual reason for threedimensionalisation) disturbance velocity component $\hat{w}$. From a numerical point of view, the effects of the imposed boundary conditions are clearly visible in this figure, namely that activity is mainly concentrated in the neighbourhood of the basic flow laminar separation bubble. Interestingly, large-scale structures analogous to those which have been identified in three-dimensional spatial DNS are present in these global linear instability results. From the point of view of identification of the global modes it is clear that the mode shown in figure 9 is distinct from those of one-dimensional theory whose essential element is their $x$-wise periodicity. In our view experimentation on linear instability in the laminar separation bubble open flow system should divert its traditional attention from the well-known scenario of existing $x$-wise periodic Tollmien-Schlichting wave-like disturbances, which experience explosive amplification after they enter the separation bubble, and consider disturbances generated within the laminar separation bubble such as that presented here. The existence and linear amplification of the latter yields inoperative any means of flow control which focusses exclusively on the former type of linear instability. Again, the first step towards identification of the global disturbances experimentally is 


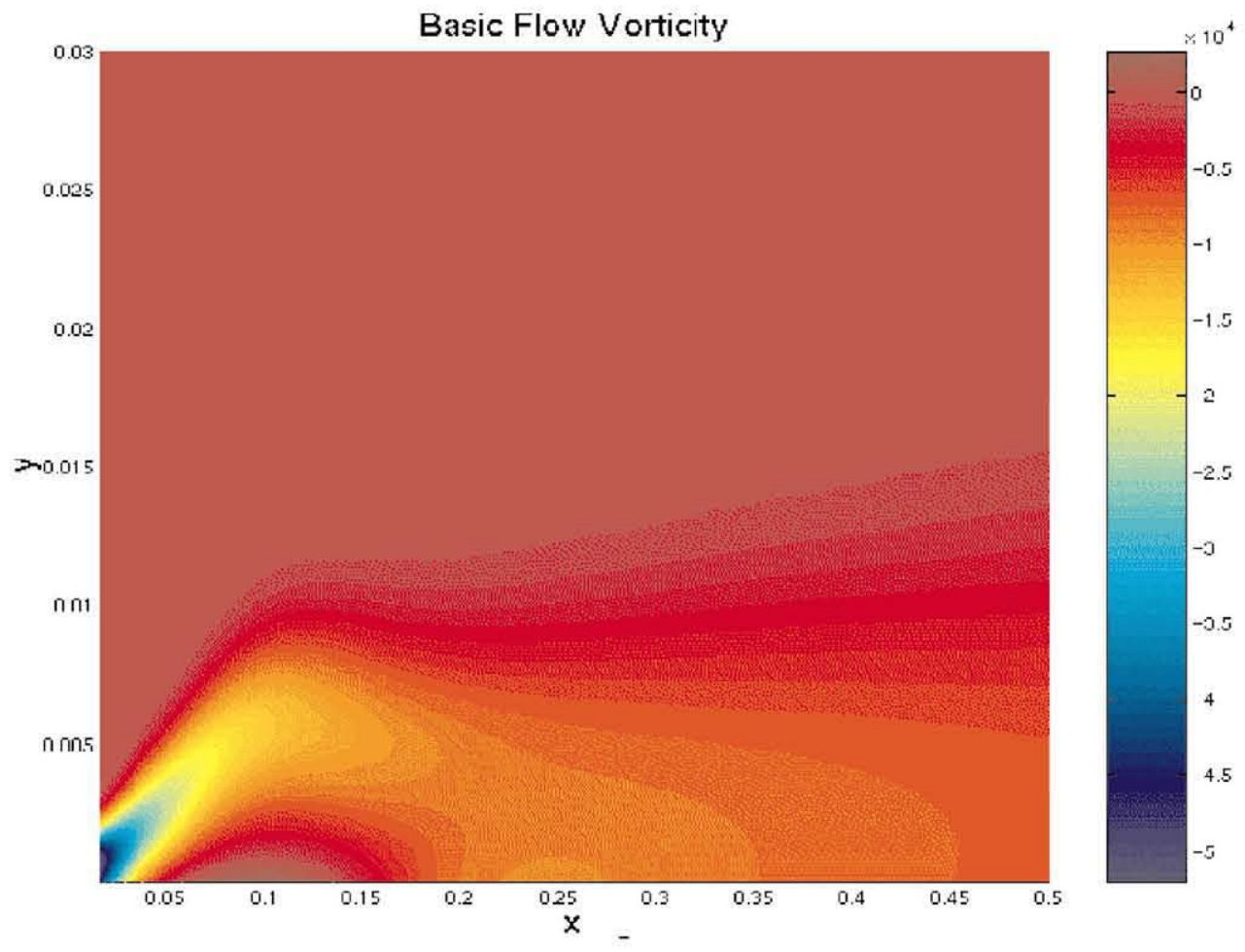

Figure 8. The two-dimensional laminar separation bubble basic flow solved by Briley [3], presented as colour-coded contour lines of the basic flow vorticity.

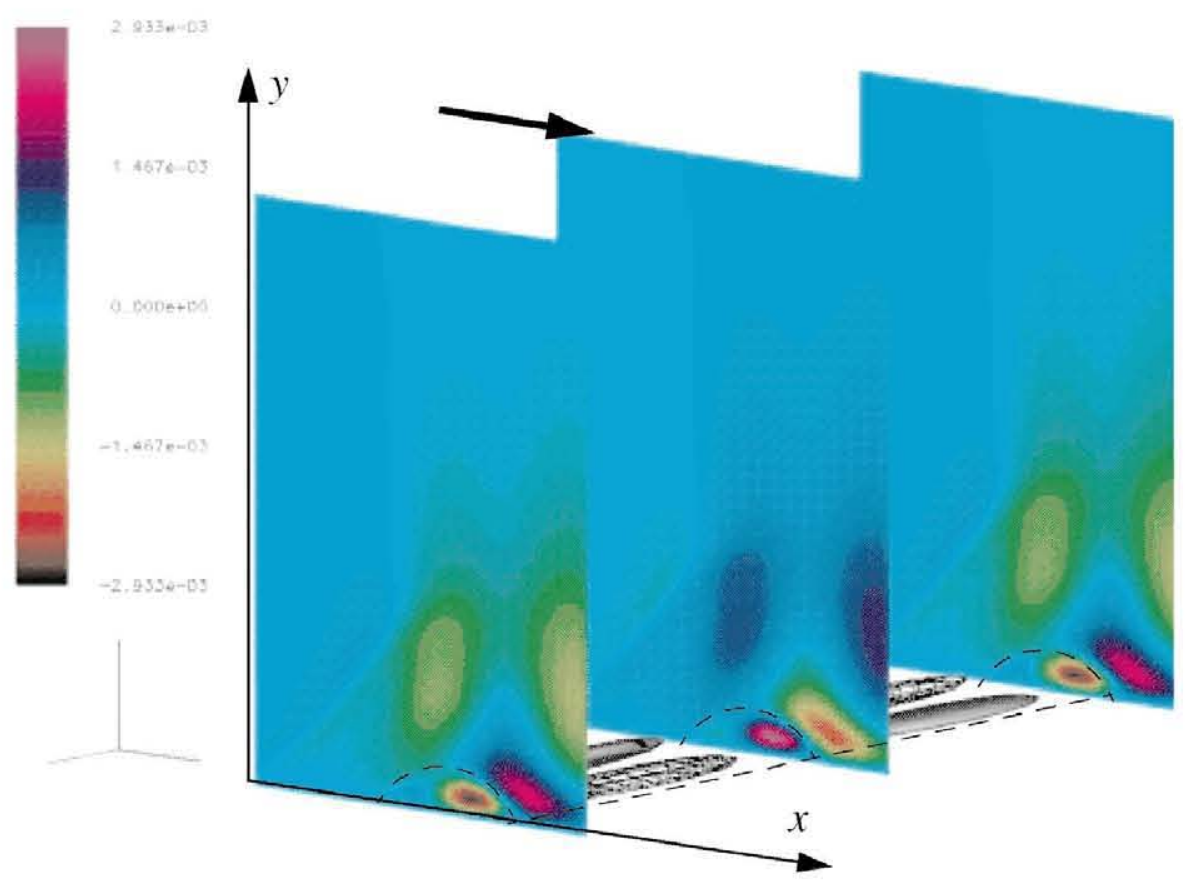

Figure 9. Perspective view of the most unstable linear global eigenmode in Briley's laminar separation bubble flow [25] presented as colour-coded contour lines of the disturbance velocity component $\hat{v}$ and isosurfaces of the disturbance velocity component $\hat{w}$ at an (arbitrary) positive level +0.02 (light-coloured surface) and its opposite (dark-coloured surface). Flow is in the direction of the arrow and the boundaries of the steady laminar separation bubble are outlined by dashed lines. 

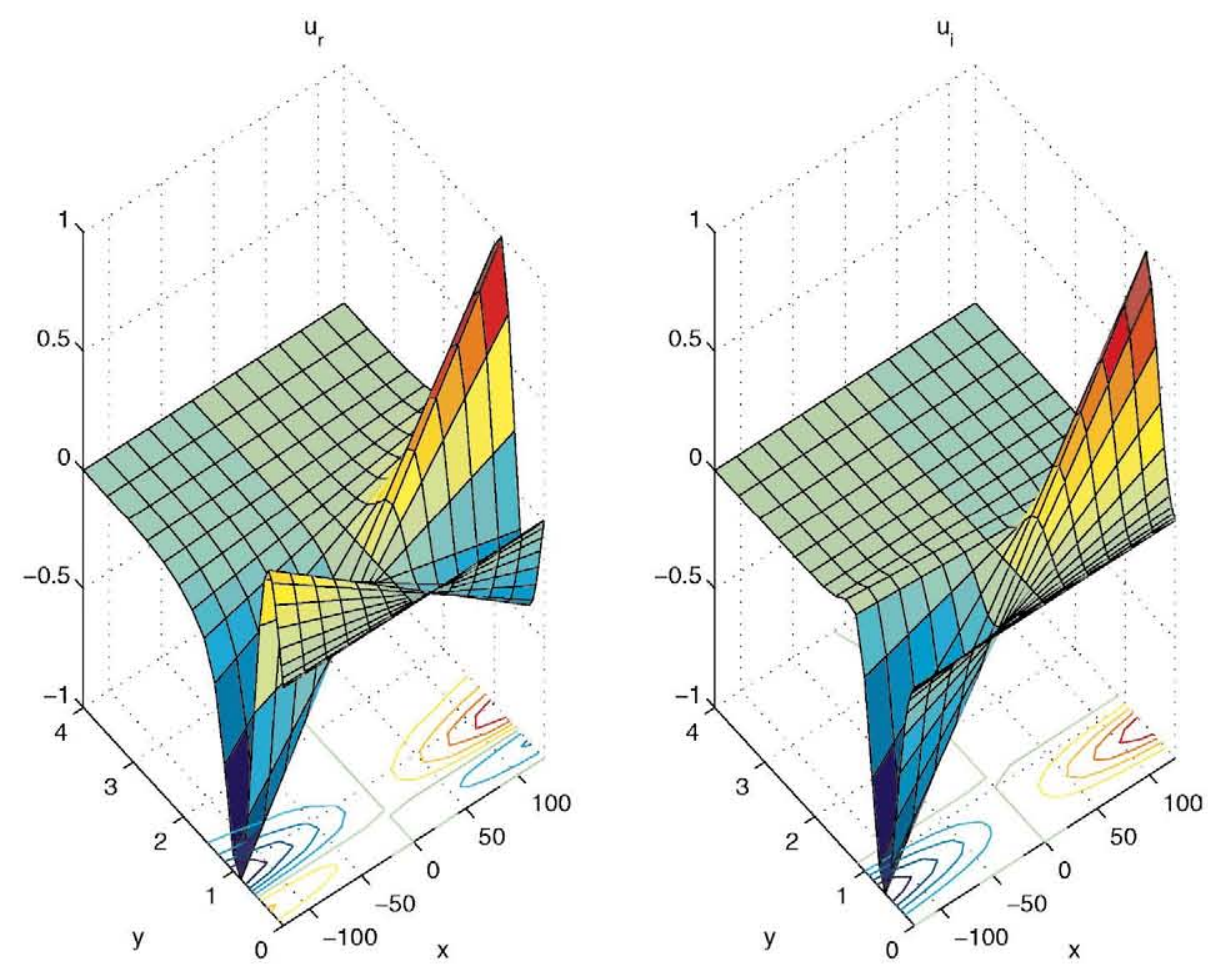

Figure 10. Streamwise disturbance velocity component of the GH mode in the incompressible infinite swept attachment-line boundary layer flow. $R e=800, \beta=0.25$.

obtaining information from a numerical solution of the partial-derivative eigenvalue problem.

\subsection{The swept attachment-line boundary layer}

This flow is one in which a number of factors have worked together in the direction of concealing unstable global linear eigenmodes despite over four decades of continuous experimentation. The new modes were discovered by solution of the partial derivative eigenvalue problem by Lin and Malik [14], their existence was confirmed by Joslin using spatial DNS [11] and their spatial structure was analysed by Theofilis [23], who also derived a one-dimensional model which extends the 1955 Görtler-Hämmerlin (GH) Ansatz to three spatial dimensions; here we briefly expose the spatial structure of the global eigenmodes. The latter are present in the flow at a certain Reynolds number and wavenumber parameters as families in which the mode postulated by Görtler and Hämmerlin and recovered numerically by Hall et al. [8] is the most unstable. The simple spatial structure of the amplitude functions of the GH mode, whose disturbance eigenvector satisfies [7,9]

$$
\begin{aligned}
& \hat{u}(x, y)=u^{\prime}(y) x, \\
& \hat{v}(x, y)=\hat{v}(y),
\end{aligned}
$$

$$
\hat{w}(x, y)=\hat{w}(y),
$$

where $x$ and $y$ respectively denote the spatial coordinates along the chordwise and wall-normal directions, permits recovery of the GH mode by a one-dimensional linear eigenvalue problem, that solved by Hall et al. [8]. In three-dimensional space the GH mode is taken to be periodic along the spanwise $z$ direction along the attachment line, such that

$$
\begin{aligned}
\tilde{u}(x, y, z, t) & =\hat{u}(x, y) E, \\
\tilde{v}(x, y, z, t) & =\hat{v}(x, y) E, \\
\tilde{w}(x, y, z, t) & =\hat{w}(x, y) E,
\end{aligned}
$$

with $E \equiv \mathrm{e}^{\mathrm{i}\{\beta z-\omega t\}}$. However, the work of Lin and Malik $[14]$ has revealed that global linear instabilities with amplitude functions other than the simple GH structure exist in this flow. Solution of the partial-derivative eigenvalue problem $[23,24]$ reveals the spatial structure of the amplitude eigenfunctions. The chordwise disturbance velocity components pertaining to the three most unstable global linear disturbances at $R e=800, \beta=0.25$ is shown in figures 10 to 12 as function of the chordwise and wallnormal spatial coordinates; an algebraic (polynomial) dependence of $\hat{u}(x, y)$ on $x$ is clearly visible in these results.

The work of Lin and Malik [14] revealed that the additional modes have frequencies which are different 

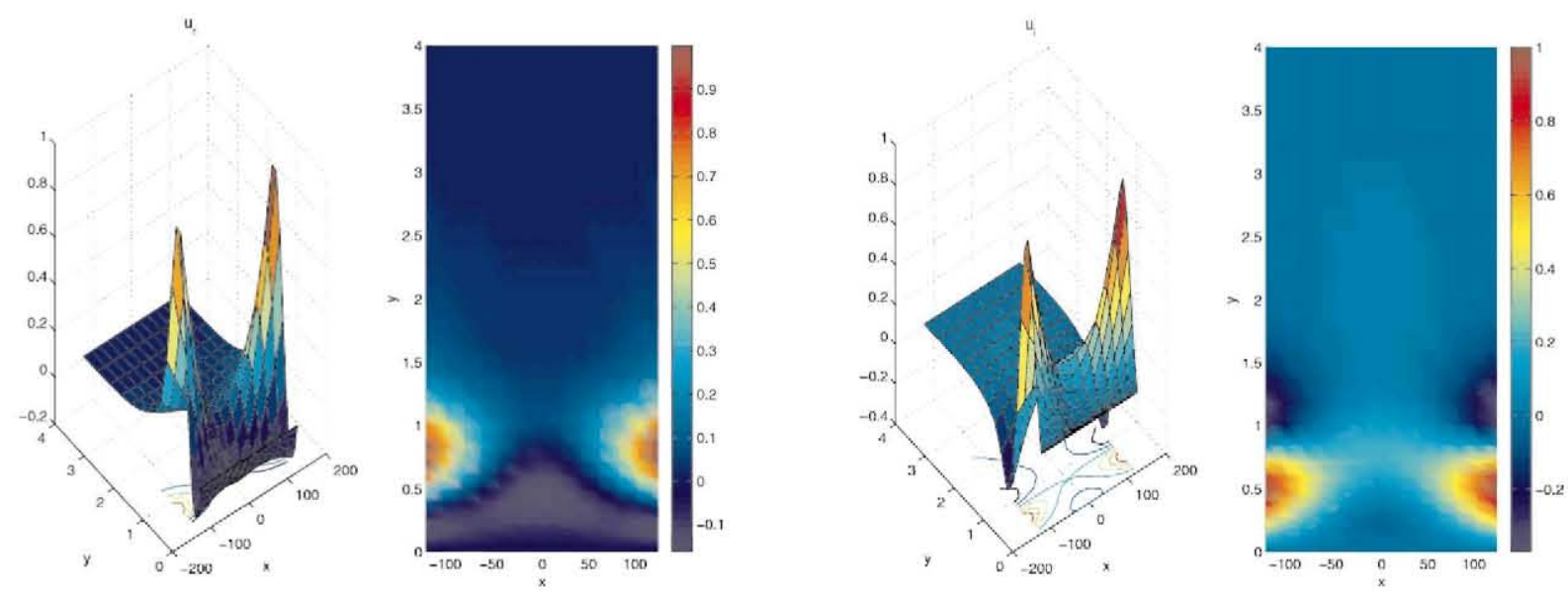

Figure 11. Real part (left) and imaginary part (right) of the streamwise disturbance velocity component of the $\mathrm{A} 1$ mode in the incompressible infinite swept attachment-line boundary layer flow. $R e=800, \beta=0.25$.
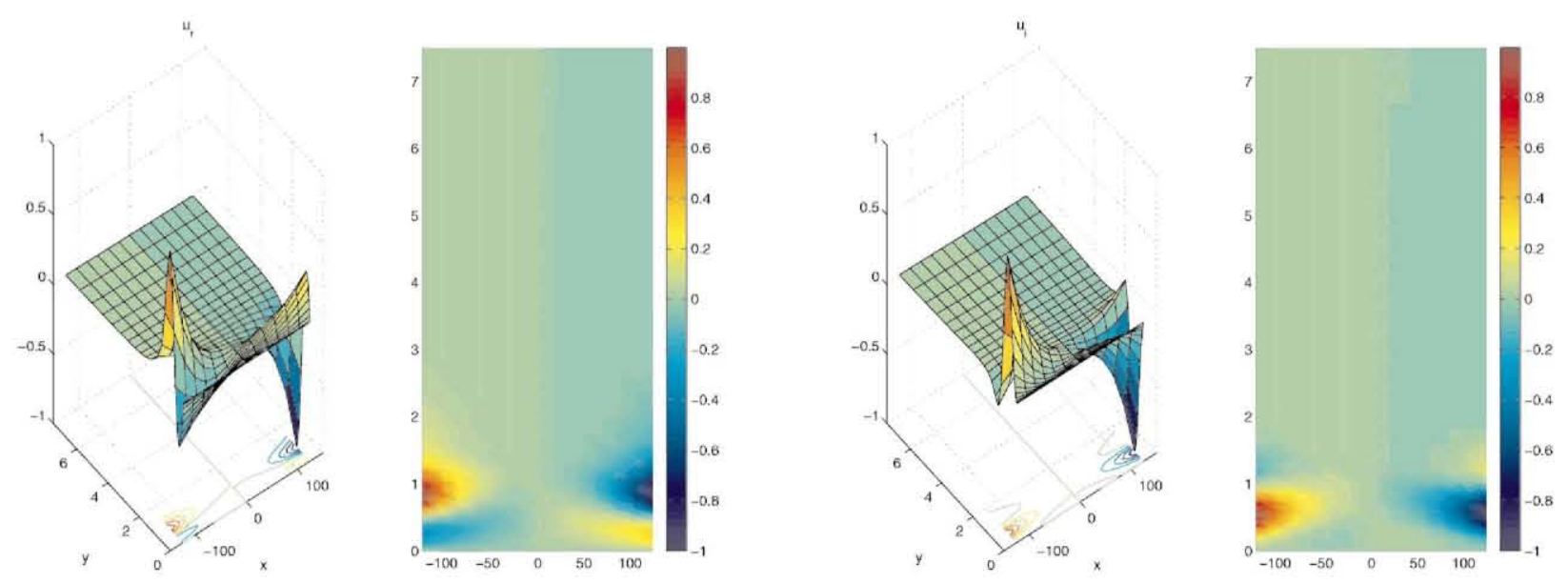

Figure 12. Real part (left) and imaginary part (right) of the streamwise disturbance velocity component of the S2 mode in the incompressible infinite swept attachment-line boundary layer flow. $R e=800, \beta=0.25$.

in relative terms to that of the GH mode by a few percent, making their identification impossible by power spectral analysis alone. The analysis of Theofilis [23] has revealed that on one hand the $y$-dependence of the new eigenmodes is identical with that of the GH mode and on the other hand the global modes may be classified into two classes of either symmetric

$$
\begin{gathered}
\hat{u}(x, y)=\sum_{m=1}^{M} x^{2 m-1} \tilde{u}_{2 m-1}(y), \\
\hat{v}(x, y)=\sum_{m=1}^{M} x^{2 m-2} \tilde{v}_{2 m-2}(y), \\
\hat{w}(x, y)=\sum_{m=1}^{M} x^{2 m-2} \tilde{w}_{2 m-2}(y),
\end{gathered}
$$

or antisymmetric

$$
\begin{aligned}
\hat{u}(x, y) & =\sum_{m=1}^{M} x^{2 m} \tilde{u}_{2 m}(y), \\
\hat{v}(x, y) & =\sum_{m=1}^{M} x^{2 m-1} \tilde{v}_{2 m-1}(y), \\
\hat{w}(x, y) & =\sum_{m=1}^{M} x^{2 m-1} \tilde{w}_{2 m-1}(y) .
\end{aligned}
$$

linear disturbances. The GH mode is a symmetric mode recovered from (13) by setting $M=1$, the first antisymmetric mode Al is that having $M=1$ in (14), followed by the second symmetric mode $\mathrm{S} 2$ which corresponds to $M=2$ in (13), the second antisymmetric mode A2 which 
corresponds to $M=2$ in (14), etc. This classification permits recovery of the partial-derivative eigenvalue problem results from an ordinary-differential-equation-based eigenvalue problem which may be solved at a negligible fraction of the cost of solving (3)-(6); details may be found in [23].

From the point of view of the theme of the present paper, the attachment-line boundary layer is a classic example of physical information being hidden or misinterpreted on account of experimental techniques which are inherently based on the assumptions of Tollmien's theory. The most common means of identification of linear instabilities, that based on power spectral density information, cannot deliver the additional to the GH eigenmodes of the flow on account of their near-identical frequencies with that of the GH mode. The more sophisticated experimental approach based on a single hot-wire transversing in the wall-normal direction will also deliver incomplete results on account of the identical $y$-dependence of the amplitude functions. One must resort to the only remaining discriminating characteristic of the eigenmodes delivered by the global linear instability analysis, namely their $x$-dependence. Use of multiple hot-wire probes (D. I. A. Poll, personal communication) offers a straightforward means for identification of the global linear instabilities (13)-(14).

\section{Conclusions}

Our objective here has been to present the spatial structure of global flow instabilities which may grow linearly upon four essentially two-dimensional incompressible laminar basic flows. In all examples presented the point was stressed that traditional means of identification of linear instabilities, deriving from the results of Tollmien's classic theory on wave-like disturbances developing upon a flat-plate boundary layer, are hardly adequate for the identification of the structures pertaining to the global eigenmodes discussed. Yet, these modes are significant on (at least) two counts. Firstly, homogeneity of physical space in the present nonparallel linear instability theory is assumed in one spatial direction alone. Consequently, the global eigenmodes form a much wider basis on which an arbitrary initial condition may be decomposed, compared with that formed by the eigenmodes of the one-dimensional Tollmien theory. Secondly, in a manner analogous to that experienced in the one-dimensional theory, once generated within the flow these global linear instabilities will grow exponentially at appropriate parameter ranges and lead laminar flow into transition and breakdown to turbulence.

Another analogy of the two-dimensional nonparallel extension of Tollmien's theory with the latter is that a feature of the global eigenmodes discovered is that their spatial structure is conditioned by the respective basic flow. Examination of the full spectrum reveals that increasingly higher modes possess increasingly more complicated structure compared with both the lower eigenmodes and the basic flow upon which they develop. This feature is reminiscent of the multi-peak structure of the Tollmien-Schlichting instabilities developing upon a Blasius boundary-layer or those in the plane channel flow. In the Tollmien theory the one-dimensional nature of the amplitude functions leaves room for (careful) single hot-wire probe measurements to be performed. This is no longer possible in the case of global linear instabilities; alternative means, based on modern global measurement techniques or developments thereof are called for. While frequency information is certainly the easiest to acquire, the critical measurement to be performed is, in our view, that of two-dimensional eigenfunctions.

The present theoretical extension of both Tollmien's theory and PSE to two resolved directions has only been made possible on account of an ever-increasing computing capacity. In the light of the significance of the new results obtained it would be highly desirable that this development be accompanied by renewed experimental efforts to identify and quantify some of the linear instability phenomena which up to the present time were erroneously classified as an unquantifiable nonlinearity.

\section{References}

[1] Barkley D., Gomes G., Henderson R.D., Threedimensional instability in flow over a backward facing step, J. Fluid. Mech (1999) (submitted).

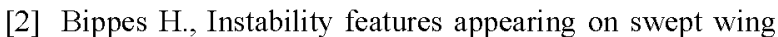
configurations, in: Arnal D., Michel R. (eds), Proceedings, Third IUTAM Symposium on Laminar-Turbulent Transition, Toulouse, 1990, pp. 419-430.

[3] Briley W.R., A numerical study of laminar separation bubbles using the Navier-Stokes equations, J. Fluid Mech. 47 (1971) 713-736.

[4] Ding Y., Kawahara M., Linear stability of incompressible flow using a mixed finite element method, J. Comput. Phys. 139 (1998) 243-273.

[5] Dovgal A.V., Kozlov V.V., Michalke A., Laminar boundary layer separation: Instability and associated phenomena, Prog. Aerospace Sci. 30 (1994) 61-94.

[6] Drazin P.G., Reid W.H., Hydrodynamic Stability, Cambridge University Press, 1981

[7] Görtler H., Dreidimensionale Instabilität der ebenen Staupunktströmung gegenüber wirbelartigen Störungen, in: Görtler H., Tollmien W., (eds), 50 Jahre Grenzschichtforschung, Vieweg und Sohn, 1955, pp. 304-314.

[8] Hall P., Malik M.R., Poll D.I.A., On the stability of an infinite swept attachment-line boundary layer, Proc. Roy. Soc. Lond. A 395 (1984) 229-245

[9] Hämmerlin G., Zur Instabilitätstheorie der ebenen Staupunktströmung, in: Görtler H., Tollmien W. (eds), 50 
Jahre Grenzschichtforschung, Vieweg und Sohn, 1955, pp. 315-327.

[10] Herbert Th., Parabolized stability equations, Ann. Rev. Fluid Mech. 29 (1997) 245-283.

[11] Joslin R.D., Simulation of three-dimensional symmetric and antisymmetric instabilities in attachment-line boundary layers, AIAA J. 34 (1996) 2432-2434.

[12] Ku H.C., Hirsch R.S., Taylor T.D., A pseudospectral method for solution of the three-dimensional incompressible Navier-Stokes equations, J. Comput. Phys. 70 (1987) $549-462$.

[13] Lin C.C., The Theory of Hydrodynamic Stability, Cambridge University Press, 1955.

[14] Lin R.-S., Malik M.R., On the stability of attachmentline boundary layers: Part 1 . the incompressible swept hiemenz flow, J. Fluid Mech. 311 (1996) 239-255.

[15] Mack L., A numerical study of the temporal eigenvalue spectrum of the Blasius boundary layer, J. Fluid Mech. 79 (1976) 497-520.

[16] Mack L., Boundary layer linear stability theory, Technical Report 709, AGARD, 1984.

[17] Nishioka M., Iida S., Ichikawa Y., An experimental investigation of the stability of plane Poiseuille flow, J. Fluid Mech. 72 (1975) 731-751.

[18] Orszag S.A., Accurate solution of the Orr-Sommerfeld stability equation, J. Fluid Mech. 50 (1971) 689-703.
[19] Ramanan N., Homsy G.M., Linear stability of lid-driven cavity flow, Phys. Fluids 6 (8) (1994) 2690-2701.

[20] Reed H.L., Saric W.S., Stability of three-dimensional boundary layers, Ann. Rev. Fluid Mech. 21 (1989) 235284

[21] Schubauer G.B., Skramstadt H.K., Laminar boundary layer oscillations and transitions on a flat plate, J. Aero. Sci. 14 (1947) 69-76.

[22] Tatsumi T., Yoshimura T., Stability of the laminar flow in a rectangular duct, J. Fluid Mech. 212 (1990) 437-449.

[23] Theofilis V., On the verification and extension of the Görtler-Hämmerlin assumption in three-dimensional incompressible swept attachment-line boundary layer flow, Technical Report IB 223-97 A 44, DLR, 1997.

[24] Theofilis V., Linear instability in two spatial dimensions in: Papailiou K.D. (ed.), Fourth European Computational Fluid Dynamics Conference ECCOMAS '98, Chichester, N. York, J. Wiley and Sons, 1998, pp. 547-552.

[25] Theofilis V., Hein S., Dallmann U.Ch., On the origins of unsteadiness and three-dimensionality in a laminar separation bubble, Philos. T. Roy. Soc. A (2000) (accepted for publication).

[26] Tollmien W., Über die Entstehung der Turbulenz, Nach. Ges. Wiss, Göttingen, 1929, pp. 21-44. 